'Unidad de Neurología

Unidad de Cardiología.

3Unidad de Hematología.

Hospital San Juan de Dios. Santiago. Chile.

Interno de $7^{\circ}$ año de Medicina. Universidad de Chile. Santiago.

Chile.

bBecario de Neurología.

Universidad de Chile. Santiago.

Chile.

Recibido el 29 de abril de 2014, aceptado el 3 de septiembre de 2014.

Correspondencia a: Carlos Guevara

Fono: 94423761 neurocrs@gmail.com

\section{Accidente cerebrovascular isquémico en pacientes con trombo intracavitario: Experiencia con tratamientos distintos en fase aguda}

\author{
GERARDO OBERREUTER ${ }^{\mathrm{a}}$, NATALIA SILVA ${ }^{\mathrm{b}}$, SHEILA CABA $^{1}$, \\ MARCELO MORALES ${ }^{2}$, ELENA NIETO $^{3}$, CARLOS GUEVARA PHD $^{1}$
}

\section{Management of ischemic strokes derived from intraventricular thrombi. Report of two cases}

The origin of $20 \%$ of ischemic strokes is a left ventricular thrombus. We report two patients with strokes originating from cardiac thrombi, treated in two different ways. A 42-year-old diabetic man admitted with a left parietal and occipital stroke. An echocardiogram showed a left ventricular thrombus. The patient was subjected to a surgical cardiac revascularization procedure and left ventricular thrombectomy. The postoperative evolution was uneventful and the patient was discharged on oral anticoagulation. On ambulatory follow up, no neurological deterioration was evidenced. A 38-year-old male admitted with an acute ischemic stroke, was subjected to thrombolysis with human plasminogen activator. An echocardiogram showed a left ventricular thrombus, considered the cause of the stroke. The patient was anticoagulated with heparin and discharged ten days after admission with oral anticoagulation. A new echocardiogram performed one month later, did not show the left ventricular thrombus.

(Rev Med Chile 2014; 142: 1200-1204)

Key words: Coronary thrombosis; Stroke; Thrombolytic therapy; Thrombectomy.
E n Chile, la principal causa de muerte se debe a patología del sistema cardiovascular $\triangle y$ corresponde a $27 \%$ de las defunciones por grandes grupos de causa de muerte. Dentro de ésta, los accidentes cerebrovasculares (ACV) corresponden a la primera causa específica de muerte, representando $9 \%$ del total de defunciones el año $2010^{1}$. Esta patología aporta además la mayor carga de enfermedad por causa específica en mayores de 74 años (AVISA) ${ }^{2}$. Del total de los $\mathrm{ACV}$, cerca de $70 \%$ corresponden a la forma isquémica $^{3}$. Entre los distintos factores que producen esta patología, la asociación con enfermedad cardiovascular está bien documentada ${ }^{4}$, estimándose que el cardioembolismo sería responsable de cerca de $20 \%$ de los casos. De este grupo, después de la fibrilación auricular, la presencia de trombo intracavitario asociada a infarto agudo al miocardio es la causa más frecuente ${ }^{5}$. Presentamos dos casos clínicos de pacientes cursando un ACV isquémico que comparten características similares en cuanto a edad, sexo y el antecedente de infarto agudo al miocardio (IAM) previo y que en el estudio de fuente embolígena se encuentra un trombo intracavitario.

\section{Caso clínico 1}

Hombre de 42 años con antecedente de diabetes mellitus tipo 2 y amputación de primer ortejo del pie derecho. Acude al servicio de urgencias 
del Hospital San Juan de Dios (HSJD) por cuadro de dos días de evolución de cefalea occipital de inicio súbito, de intensidad 8/10, asociado a fono-fotofobia, visión borrosa bilateral y vértigo. Al examen físico se observa consciente, orientado témporo-espacialmente, hemodinámicamente estable, pupilas isocóricas, reflejo fotomotor directo y consensual conservados, oculomotilidad sin alteraciones, destacando una hemianopsia homónima derecha. La tomografía computada (TC) de cerebro sin contraste mostraba lesiones hipodensas parietooccipital izquierda y occipital derecha, siendo esta última compatible con un quiste aracnoidal (Figura 1). Un electrocardiograma mostró signos de infarto antiguo. Se hospitalizó para estudio con diagnóstico de ACV isquémico parieto-occipital izquierdo. Al quinto día del inicio de sus síntomas, un ecocardiograma transtorácico mostró disfunción sisto-diastólica con fracción de eyección de $40 \%$, leve dilatación auricular izquierda y masa pediculada intraventricular izquierda de $7 \times 4 \mathrm{~cm}$, sugerente de trombo intracavitario (Figura 2). Se inició anticoagulación con bomba de infusión continua (BIC) de heparina no fraccionada (HNF) y se decidió realizar una trombec-

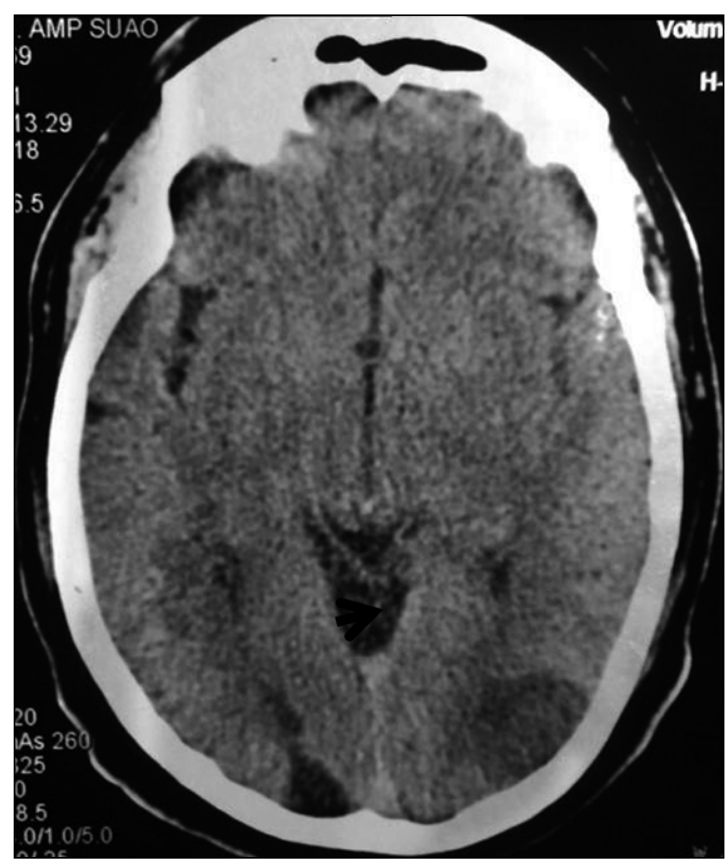

Figura 1. TC cerebro sin contraste que muestra hipodensidad parietooccipital izquierda, sugerente de infarto en dicha zona (flecha). tomía por elevado riesgo de desprendimiento del trombo y obstrucción de válvula aórtica. En el estudio preoperatorio destacaba coronariografía que evidenciaba arterias coronarias con ateromatosis moderada, no estenosante y disección de coronaria derecha a nivel medio distal. El mismo quinto día se realizó cirugía de revascularización miocárdica más trombectomía en región ínfero-apical de ventrículo izquierdo con circulación extracorpórea (CEC), bajo heparinización endovenosa a $3 \mathrm{mg} /$ $\mathrm{kg}$. La biopsia del trombo extraído concluyó un coágulo en organización y el cultivo de la muestra resultó negativo.

El paciente evolucionó favorablemente, sin signos de transformación hemorrágica en TC cerebral de control. Fue dado de alta al cuarto día postoperatorio con anticoagulación oral, sin evidencia de deterioro neurológico en controles ambulatorios posteriores.

\section{Caso clínico 2}

Hombre de 38 años, profesor de computación, con antecedente de infarto agudo al miocardio en el año 2011, ocasión en la que se le realizó una angioplastia con endoprótesis vascular no medicada en arteria coronaria descendente anterior, con posteriores controles ambulatorios abandonados. Tiene además antecedente de tabaquismo activo de 10 cigarrillos/día. Acudió al servicio de urgencias del HSJD por presentación

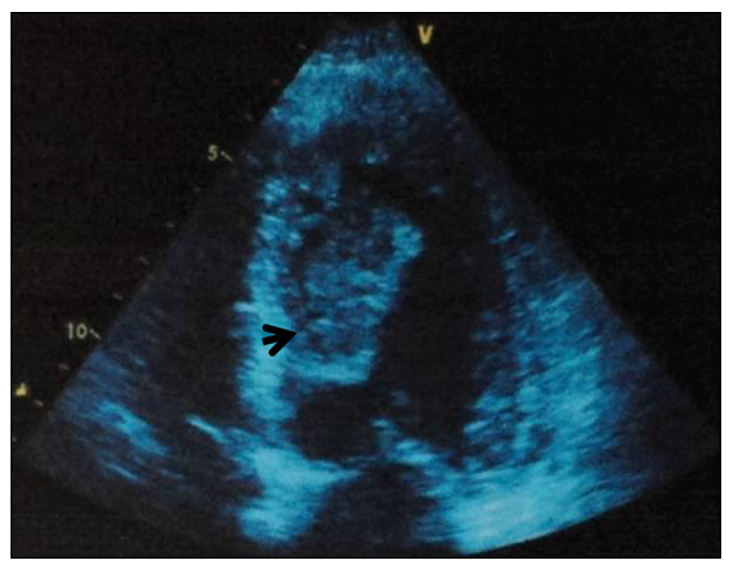

Figura 2. Ecocardiograma transtorácico 2D donde se aprecia masa pediculada intracavitaria de $7 \times 4 \mathrm{~cm}$ en ventrículo izquierdo (flecha). 
súbita de desviación de comisura labial izquierda y pérdida de fuerza del hemicuerpo izquierdo. Ingresó a urgencias con una hora de evolución desde el inicio de los síntomas. Al examen neurológico de ingreso destacaba desorientación témporo-espacial, escala de Glasgow 14, afasia motora severa, hemiplejia facio-braquio-crural izquierda y hemianopsia homónima izquierda. En la escala del National Institute of Health Stroke Scale (NIHSS) obtuvo puntuación de 19. La TC de cerebro sin contraste no evidenció lesiones agudas ni antiguas. Se diagnosticó un accidente cerebro vascular $(\mathrm{ACV})$ isquémico agudo en evolución y se activó protocolo de trombolisis con activador del plasminógeno humano (rt-PA). Dado que el paciente cumplió criterios de inclusión para el procedimiento, se inició trombolisis a una hora y diez y siete minutos de iniciados los síntomas. Se estimó un peso de $70 \mathrm{~kg}$, administrándose 6,3 mg en bolo y 56,7 mg en infusión en una hora. Durante el procedimiento destacaba tendencia a la hipotensión hasta presión arterial media (PAM) de $78 \mathrm{mmHg}$ que se manejó con suero fisiológico endovenoso, lográndose PAM cercanas a 100 $\mathrm{mmHg}$, sin mayores complicaciones.

Evolucionó favorablemente: evaluado con la escala del NIHSS se observó una baja desde 19 al inicio de la trombolisis, a 15 a los sesenta minutos y 3 a los noventa.

Al día siguiente se observó en GCS 15, con paresia mínima en hemicara inferior izquierda, sin otras focalidades ni alteraciones neurológicas. Se realizó TC cerebral de control que evidenció áreas hipodensas fronto-parietales derechas con signos de edema, sin signos de transformación hemorrágica (Figura 3). Ecocardiograma transtorácico detectó un trombo intra-cavitario de 1,6 $\mathrm{cm} \times 2,3 \mathrm{~cm}$ (Figura 4), que fue considerado como

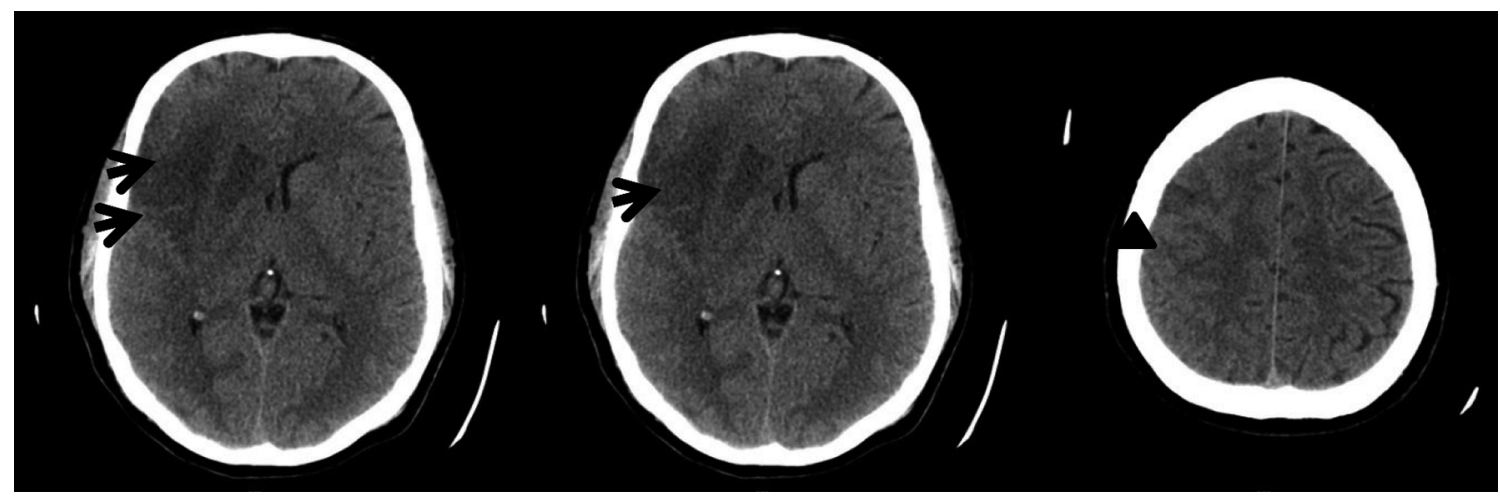

Figura 3. Tomografía computada de cerebro, tomada a las veinte y cuatro horas de iniciados los síntomas. Se observan zonas hipodensas fronto-parietales derechas (flechas), sugerentes de áreas necróticas y disminución de surcos y giros, sugerente de edema (triángulo).

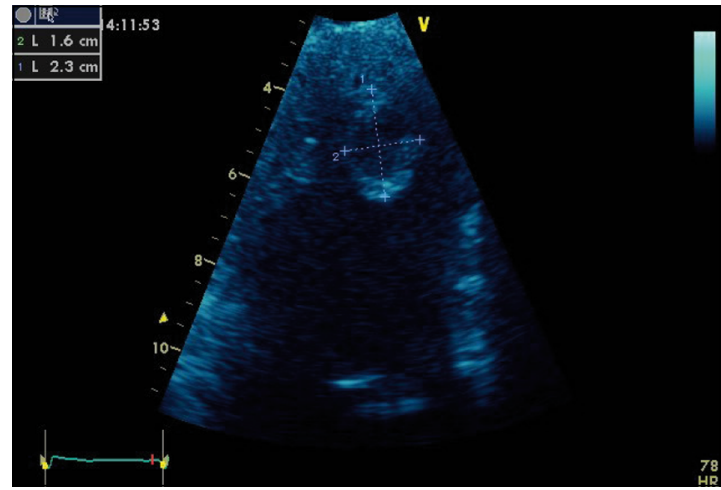

Figura 4. Trombo intracavitario en ventrículo izquierdo de $1,6 \mathrm{~cm} \times 2,3 \mathrm{~cm}$.

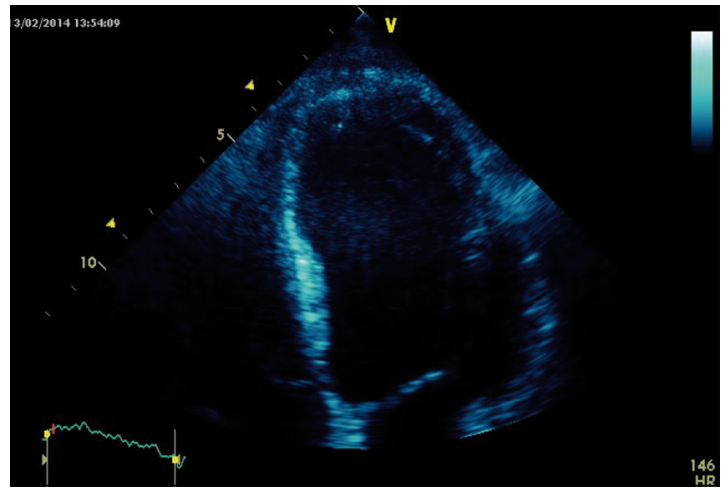

Figura 5. Ventrículo izquierdo un mes después de iniciada la terapia anticoagulante, donde destaca la ausencia del trombo. 
fuente del émbolo causante del ACV isquémico. A los 5 días de ocurrido el ACV, se inició heparina de bajo peso molecular endovenosa con posterior traslape a anticoagulantes orales. Es dado de alta al décimo día con acenocumarol. Estudio de hipercoagulabilidad heredada mostró valores normales de proteína $\mathrm{S}$, proteína $\mathrm{C}$, homocisteinemia, antitrombina III y ausencia del factor $\mathrm{V}$ de Leiden (mutación R506Q) y de la mutación G20210A del gen de la protrombina. En control con ecocardiograma transtorácico, un mes después, ya no se observaba el trombo en el ventrículo izquierdo (Figura 5). El paciente evolucionó con mínima paresia facial izquierda como secuela del evento isquémico (escala de Rankin modificada de 1).

\section{Discusión}

La literatura señala que la incidencia de ACV isquémico en pacientes que han sufrido un IAM oscila entre $0,84 \%-1,14 \%$. Los factores de riesgo más relacionados entre ambos eventos son edad avanzada, clase funcional de Killip, extensión del infarto y ubicación en pared anterior ${ }^{6-8}$. Destacamos un estudio de 20.768 pacientes hospitalizados por IAM, donde la incidencia de ACV isquémicos o hemorrágicos entre pacientes que recibieron heparina profiláctica y quienes no, no mostró diferencias significativas ${ }^{6}$. Así los pacientes que han sufrido un IAM debieran ser informados sobre su riesgo de presentar un evento cerebrovascular posteriormente, de manera que adopten un riguroso control cardiovascular y también sepan reconocer los síntomas de un ACV y así acudir oportunamente a un servicio de urgencias.

Una de las complicaciones más temidas en el curso de un ACV isquémico es la hemorragia intracerebral sintomática (HICS), la que puede darse en presencia de hipertensión arterial mal controlada, durante el uso de fibrinolíticos o terapia anticoagulante con heparinas ${ }^{9,10}$. El primer caso expuesto presenta hipoquinesia y fracción de eyección disminuida asociada a un trombo intraventricular izquierdo gigante con riesgo de desprendimiento y obstrucción de válvula aórtica. Estudios sugieren que la terapia anticoagulante en pacientes con falla cardiaca y trombos gigantes móviles, no previene su fragmentación y embolización $^{11}$, por lo que la remoción quirúrgica se reserva para estos casos, como fue el caso de nuestro primer caso clínico ${ }^{12}$. El uso de circulación extracorpórea tradicional con heparinización produjo un potencial riesgo de transformación hemorrágica, dado el infarto extenso en quinto día de evolución. Estudios aleatorios no han sido concluyentes con respecto a la mejor opción en cuanto a costo versus beneficios al comparar los circuitos con CEC heparinizados sobre CEC tradicional ${ }^{13}$, siendo este último circuito es el que se encontraba disponible en nuestro hospital.

Con respecto al segundo caso clínico, el uso de rt-PA para fibrinólisis en casos de ACV isquémicos agudos tiene una efectividad conocida en cuanto a recuperar la independencia del paciente y un margen de seguridad aceptable si se realiza según protocolos establecidos ${ }^{14,15}$. La evolución clínica favorable y la disolución del trombo en este paciente con un tratamiento médico ( $\mathrm{y}$ no quirúrgico como en el primer caso) demostraron que la decisión de iniciar heparina fue la adecuada. Pese a que en los ACV isquémicos con una fuente embolígena no existe una normativa clara sobre cuándo iniciar tratamiento anticoagulante, la regla empírica de iniciar anticoagulación al sexto día en infartos cerebrales medianos resultó útil en este caso.

Se conoce que los ACV isquémicos de etiología cardioembólica son severos, que están asociados a un mayor déficit neurológico y a una mayor discapacidad a los seis meses de ocurrido el evento en comparación con los ACV no cardioembólicos. En ambos casos expuestos, la decisión de anticoagular con heparina en presencia de infarto cerebral de mediana cuantía fue una decisión que se tomó en conjunto con hematología, cardiología y neurología de nuestro hospital, evaluando los riesgos y beneficios entre anticoagular y no hacerlo.

\section{Conclusión}

Se presentaron a dos pacientes jóvenes cursando un ACV isquémico asociado a un IAM previo con trombos cardiaco intra-cavitario. El manejo inicial fue empírico dado la ausencia de protocolos validados en estos casos y difirió en consideración del tiempo de evolución de infarto cerebral y del tamaño del trombo cardiaco. Ambos casos evolucionaron favorablemente, uno con enfoque quirúrgico y el otro con tratamiento médico seguidos por el uso de heparina en la fase aguda del 
ACV isquémico. Se hace así necesario protocolizar el manejo de los pacientes con trombos intracardiacos cursando con una isquemia cerebral aguda, considerando el tamaño del trombo y la extensión del infarto cerebral en curso.

\section{Referencias}

1. Ministerio de Salud de Chile. Indicadores básicos de salud y diez primeras causas de muerte. Chile 2000 a 2010. Departamento de estadísticas e información de salud. URLhttp://www.deis.cl

2. Ministerio de Salud de Chile. Informe final estudio de Carga de Enfermedad y Carga Atribuible 2007. http:// www.deis.cl [Consultado el 15 de junio de 2012].

3. Lavados P, Sacks C, Prina L, Escobar A, Tossi C, Araya F et al. Incidence, 30-day case-fatality rate, and prognosis of stroke in Iquique, Chile: a 2-year community-based prospective study (PISCIS project). Lancet 2005; 365: 2206-15.

4. Baena J, Álvarez B, Piñol P, Martín R, Nicolau M, Aiteg A, et al. Asociación entre la agrupación (clustering) de factores de riesgo cardiovascular y el riesgo de enfermedad cardiovascular. Rev Esp Salud Pública [online] 2002; vol 76, n. 1 [citado 2014-02-09], pp. 07. http:// scielo.isciii.es/scielo.php?script=sci_arttext\&pid=S113557272002000100002\&lng=es\&nrm=iso $>$

5. Bogousslavsky J, Cachin C, Regli F, Despland Pa, Van Melle G, Kapenberg L, et al. Cardiac sources of embolism and cerebral infarction. Clinical consequences and vascular concomitants: The Lausanne Stroke Registry. Neurology 1991; 41: 855-959.

6. Pietro A, Grazia M, Santoro E, White H, Van de Werf F, Tognoni G, et al. The risk of stroke in patients with acute myocardial infarction after thrombolytic and antithrombotic treatment. The New England Journal of Medicine. Volume 327. July 2, 1992.

7. Maggioni A, Franzosi M, Farina M, Santoro E, Celani M, Ricci S, et al. Cerebrovascular events after myocardial infarction: analysis of the GISSI trial. BMJ 1991; 302 (6790): 1428-31.

8. Thompson P, Robinson J. Stroke after acute myocardial infarction: relation to infarct size. Br Med J 1978; 2 (6135): 457-9.

9. Navarrete, P. Manejo inicial del ictus isquémico agudo. Med Intensiva 2008; 32 (9): 431-43.

10. Restrepo J. Utilidad de la heparina no fraccionada y de bajo peso molecular en el tratamiento del ataque cerebro vascular isquémico agudo de origen cardioembólico. Acta Neurol Colomb 2010; 26: 61-7.

11. Leeper N, Gupta A, Schnittger I, Wu J. Clinical dilemmas in treating left ventricular thrombus. Int J Cardiol 2007; 114 (3): 118-9.

12. Nili M, Deviri E, Jortner R, Strasberg B, Levy M. Surgical removal of a mobile, pedunculated left ventricular thrombus: report of 4 cases. Ann Thorac Surg 1988; 46 (4): 396-400.

13. Charles W, Christopher A, Joseph E. Cardiopulmonary Bypass Management and Neurologic Outcomes: An Evidence-Based Appraisal of Current Practices. Anesth Analg 2006; 103: 21-37.

14. Buchan A, Barber P, Newcommon N, Karbalai H, Demchuk, Hoyte KM, et al. Effectiveness of t-PA in acute ischemic stroke: Outcome relates to appropriateness. Neurology 2000; 54: 679.

15. Araújo D, Teich V, Passos R, Martins, S. Análisis de Costo-Efectividad de la Trombólisis con Alteplase en el Accidente Vascular Cerebral. Arq Bras Cardiol 2010; 95 (1) : 12-20. 